\title{
Resistivity estimation for a row of potato starches of domestic collection
}

\author{
L. Gvozdeva*, V. Khlestkin \\ Institute of Cytology and Genetics SB RAS, Novosibirsk, Russia \\ *e-mail: ungersy@mail.ru
}

Key words: diabetes, genome-wide association studies, potato starch, resistant starch

\begin{abstract}
Motivation and Aim: Production of starch with desired characteristics is an important and urgent task related to dietetic food (resistant starch) and (bio)chemical processing on an industrial scale. Resistant to digestion starch passes through small intestine, remaining intact, and then enters large intestine and serves as an excellent nutrient medium for beneficial local microflora. In contrast to conventional starch, the resistive one increases sensitivity of body cells to insulin and lowers the level of glucose in a blood $[1,2]$. The results of the work will make possible to choose more accurately a healthy diet for prevention and/or control of a number of diseases (e.g. diabetes) as well as more efficient use of starch in industrial processing.
\end{abstract}

Methods and Algorithms: We isolated starch from different varieties of potato mainly of Russian origin. Starch sample was subjected to digestion by $\alpha$-amylase for $16 \mathrm{~h}$ at $37{ }^{\circ} \mathrm{C}$. Precipitate obtained was split by glucosidase for $1 \mathrm{~h}$ at $60{ }^{\circ} \mathrm{C}$. We measured the amount of glucose formed with a test-kit «Glucose Olveks»at a wavelength of $500 \mathrm{~nm}$ and calculated the amount of resistive starch as a percentage of the total amount of starch taken for analysis.

Results: 90 varieties were investigated for the content of resistive raw starch. The average content of resistive starch was $70 \%$ of the total amount of starch. The contrast forms have been identified and will be used at further stages for the genome-wide association studies. That will allow identification of loci and associated DNA-markers associated with starch resistance to digestion in the upper sections of the gastrointestinal tract.

Conclusion: The first comprehensive study of the starch resistance of domestic potato varieties is performs. The data obtained will be used for associations search after genotyping. Thus, it is possible to quickly select breeding varieties rich in resistive or digestible starch that can be used in the treatment/prevention of a number of diseases (related to the digestibility of starch) or to increase the efficiency of industrial processing of starch.

Acknowledgements: The work supported by a grant from the RFBR (No. 17-29-08006). Samples of potatoes were obtained from the collection of the Genetic Control Center «GenAgro» (IC\&G SB RAS).

\section{References}

1. Robertson D. et al. (2005) Insulin-sensitizing effects of dietary resistant starch and effects on skeletal muscle and adipose tissue metabolism. American Society for Clinical Nutrition.

2. Shen R.L., Zhang W.J., Dong L.J. (2016) Preparation, structural characteristics and digestibility of resistant starches from highland barley, oats and buckwheat starches. Journal Food Nutrition Research. 55(4):303-312. 